Discussion Papers of the

Max Planck Institute for

Research on Collective Goods

Bonn 2018/15

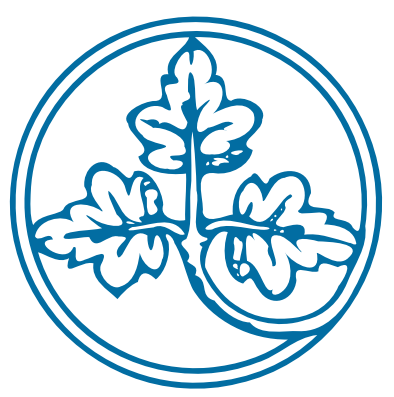

Gain-Loss Framing in Interdependent Choice

Susann Fiedler

Adrian Hillenbrand

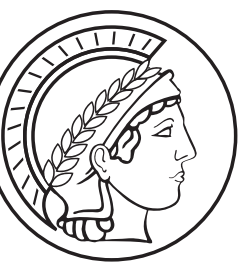




\title{
Gain-Loss Framing in Interdependent Choice
}

\author{
Susann Fiedler / Adrian Hillenbrand
}

December 2018 


\title{
Gain-Loss Framing in Interdependent Choice*
}

\author{
Susann Fiedler† Adrian Hillenbrand ${ }^{\ddagger}$
}

October 16, 2018

\begin{abstract}
Framing influences choice. However, little is known about the underlying mechanisms behind framing effects. We study gain-loss framing in binary modified dictator games. Subjects choose the selfish option more often in the loss frame compared to the gain frame. Recording visual fixations with eye-tracking, we find that dictators focus more on their own outcomes when facing losses. This suggests that losses to the own outcome are weighted more than losses to another player.
\end{abstract}

\footnotetext{
*Financial support by the Max Planck Society is greatly acknowledged.

${ }^{\dagger}$ Max Planck Institute for Research on Collective Goods, Kurt-Schumacher-Str. 10, 53113 Bonn, Germany. E-mail: fiedler@coll.mpg.de.

${ }^{\ddagger}$ Max Planck Institute for Research on Collective Goods, Kurt-Schumacher-Str. 10, 53113 Bonn, Germany. E-mail: hillenbrand@coll.mpg.de.
} 


\section{Introduction}

Choices are influenced by framing. Casual observations as well as numerous studies are in line with that statement. Framing is also a powerful tool which is regularly and intentionally used in political speeches, advertisement and health care or donation campaigns. Changing the perceived context of a situation, simple relabeling of decisions or the description of outcomes is shown to have a huge impact on human decision makers.

The frame which is studied most prominently in economics is the gainloss frame. People react differently to situations framed as a loss compared to situations framed as gains. This effect has been introduced to the economic literature in the seminal study by Tversky and Kahneman (1981) when comparing choices over lives lost compared to lives saved. Further examples are demonstrated by the fact that people are more risk-loving when facing losses compared to facing gains (Kahneman and Tversky, 1984; Tversky and Kahneman, 1991) and the endowment effect (Kahneman et al, 1990) with loss-aversion and reference-dependent preferences as the main driving factor (Kahneman and Tversky, 1979).

Importantly, being prone to framing effects does not only have consequences for independent decisions. Often decision maker's choices directly or indirectly impact the well-being of others. Evidence shows that even when decision makers take the outcome for others into account, the extent to which they do so is influenced by framing (e.g., Brekke et al, 2012; Dariel, 2013; Engel and Rand, 2014). This effect might be driven by different mental representations of the situation and with that the understanding of "what is the right thing to do". Alternatively, decision makers facing losses could be occupied with themselves while decision makers facing gains 
could have more cognitive resources at their disposal to take others' welfare into account.

Testing the framing effect in the context of interdependent choice, this paper investigates these processes by analyzing the information search and integration processes in binary modified dictator games.

For economic theory framing poses a fundamental challenge. When choices are not consistent but influenced by framing, the common revealed preference attempt is potentially misleading. This makes predictions challenging. Specifically, a decision maker's behavior as observed in one frame does not necessarily translate into similar behavior in a different frame. In behavioral economics, frames are mainly understood and modeled as a variation in the reference point (Tversky and Kahneman, 1981; Amos Tversky, 1986) and as a factor influencing beliefs (Dufwenberg et al, 2011; Ellingsen et al, 2012). A general framework is given by Salant and Rubinstein (2008) and Bernheim and Rangel (2007). They, among others, suggest that economic models should take "ancillary conditions" like frames into account to allow for welfare analysis encompassing non-standard decision makers.

Salant and Rubinstein (2008) describe a frame as "[...] observable information, other than the set of feasible alternatives, which is irrelevant in the rational assessment of the alternatives but nonetheless affects behavior". The effects of framing on choice and judgment behavior have been demonstrated in individual and interdependent decision situations various times, but exactly how framing affects behavior, i.e., how the mental representation of a situation changes, still remains unclear. So far, economists mainly focus on outcome-based models with choice as the main observational variable. It is recognized that in order to understand decision mak- 
ing and its influential factors completely, this might not be enough (e.g., Krajbich et al, 2014; Krajbich and Dean, 2015). When the aim is to develop a theory of economic decision making capable of predicting choices also in different, previously unobserved frames, a clear understanding of the underlying cognitive mechanism, i.e., the decision making process is important.

In order to study these questions, we conducted a lab experiment. Subjects play multiple modified-dictator games (Andreoni and Miller, 2002) where they decide between an own-outcome maximizing (selfish) option and an other-outcome maximizing (altruistic) option. In a between-subjects design, decisions are either framed as a gain (GAIN) or as a loss (LOSS). We use eye-tracking to record visual fixations of subjects (Lahey and Oxley, 2016).

We use eye-tracking as a source of complementary data to gain insights into the underlying mechanisms of choices. In particular, eye-fixations as an unobtrusive measure can provide important information about the weight (or importance) given to the different types of information (e.g., own outcome, recipient's outcome) during the decision making process (see, e.g., Armel et al, 2008; Krajbich et al, 2010; Krajbich and Rangel, 2011). Eyetracking is unobtrusive in that we can gain insights into the motives of decision makers without actively changing the decision environment which in itself could influence decision making. Various studies utilize eye-tracking in the context of economic decision making. These papers shed light on fairness motivations (Arieli et al, 2009), decisions under uncertainty (Arieli et al, 2011), truth-telling (Wang et al, 2010), as well as behavior in social dilemmas (Fiedler et al, 2013), consumer choice (Balcombe et al, 2017; 
Krucien et al, 2017; Reutskaja et al, 2011) and strategic interaction (Chen et al, 2018; Devetag et al, 2015; Polonio et al, 2015).

In recent years the interest in understanding the underlying mechanisms behind choices is rapidly increasing (e.g., Dohmen et al, 2011; Fehr and Rangel, 2011; Krajbich et al, 2010, 2014). With the advancements in neuroeconomics and cognitive psychology, new tools and a better understanding of how to interpret process data such as eye-movements and decision times are available (Lahey and Oxley, 2016). Studying framing effects is particularly helpful. Framing produces comparable situations which are identical from an outcome perspective. This way, every change in behavior must be a result of a different perception of the situation, i.e., a change on the process level because all other environmental features are kept constant (Levin et al, 2014).

The results of the study at hand provide evidence for a clear framing effect. Dictators facing gains choose the altruistic option more often (55\%) than subjects facing losses $(38 \%)$ in situations where the dictator has a higher income than the recipient. This difference between frames is not found in situations in which the dictator has a lower income than the recipient. Specifically interesting in light of these choice patterns is the respective change in the observed attention distribution. We find a strong difference between frames concerning subject's information search behavior. Subjects in LOSS focus more on their own income compared to subjects in GAIN.

In order to structure our results we use a behavioral model, namely Reference Dependent Altruism (Breitmoser and Tan, 2014, 2013) and interpret it as a process model. We show that the model and altruism parameters 
elicited in an online pre-test fit the choice data and process data quite well. The altruism parameters strongly correlate with relative attentional weighting of selfish and other-regarding information.

The main aim of the paper is to improve the understanding of framing effects in interdependent situations. With this paper we provide a first step towards a better understanding of how exactly frames influence decision making. Our results suggests that, when facing losses, subjects put more weight on losses to their own outcome compared to losses of the other subjects. In other words, loss aversion seems to be stronger in the ownoutcome domain compared to the other-outcome domain.

The remaining paper is structured as follows. In Section 2 we discuss further literature. We develop theoretical predictions for our study in Section 3. Section 4 describes the experimental setup. In Section 5 we present the results and discuss them in Section 6.

\section{Further Literature}

\subsection{Gain-loss framing in interdependent situations}

In line with our results multiple experiments have presented evidence showing that people's response when confronted with gains versus losses varies profoundly. Already the influential work by Kahneman and Tversky (1979) showed that losses have a bigger influence than gains of the same size (see for a more recent overview Baumeister et al, 2001). This effect has been observed in a wide range of situations and contexts alike, but mainly in the area of risky decision making (Kühberger, 1998). See Camerer and Loewenstein (2004) for an overview of loss aversion in the field, but see 
Novemsky and Kahneman (2005) for the boundaries of loss aversion.

With respect to interdependent social decisions the literature shows mixed results. In Antinyan (2014), subjects play a dictator game in which the dictator and the recipient experience a loss before making a decision. They do not find significant differences in average allocation behavior between loss and gain decisions but in the loss treatment more equal offers are observed. Buchan et al (2005) use a ultimatum game in which decisions are made about sharing a loss and sharing a gain. They find that

offers are higher when facing losses than when facings gains. Fitting to this result Zhou and Wu (2011) find in a similar setting that non-fair offers were perceived less fair in the loss domain and got rejected more often compared to the gain domain (but see Leliveld et al, 2009). In contrast to these results, further studies show that individuals in a loss condition are more own-outcome oriented (De Dreu et al, 1994; De Dreu, 1996) more individualistic (Poppe and Valkenberg, 2003) and more prone to unethical behavior (Kern and Chugh, 2009) than individuals in the gain condition. Of these papers the experimental design in Poppe and Valkenberg (2003) is most similar to ours. They map decisions of subjects to social value orientations, while we focus on the overall share of altruistic decision and on explaining effects on choices with the help of eye-gaze data.

\subsection{Process investigations on gains and losses}

Process investigations of framing effects are rare in the literature and almost exclusively concern individual decision making without any consequences for others. Further, most of these studies focus on response times.

In early studies Liebrand and McClintock (1988) and Dehue et al (1993) 
present evidence from decision time investigations of simple allocation tasks that cooperative decision makers need more time to decide about losses in comparison to gains. In general, this is understood as a consequence of loss-aversion and consequently a higher weighting of losses compared to gains. Yechiam and Hochman (2013a) suggest an alternative explanation: losses invoke an "attentional effect leading to increased sensitivity to task incentives" (Yechiam and Hochman, 2013a,b). Another paper dealing with the attentional effect is by Baumeister et al (2001). They argue that bad events (e.g., losses) should capture more attention compared to good events (e.g., gains). Alternatively, this might be due to a negative mood raised by a negative frame inducing an increase in cognitive effort in the domain of losses (Kuo et al, 2009). More broadly, many studies showed that losses have a stronger effect on physiological arousal (e.g., heart rate and pupil dilations) compared to gains (Satterthwaite et al, 2007; Hochman et al, 2010; Hochman and Yechiam, 2011).

In contrast to these studies, we do not find a direct effect of the framing manipulation on decision times. Further, we mainly focus on the relative attention to specific information.

\subsection{Assumptions about the relationship between gaze behavior and the cognitive process}

Through technological progress in the area of gaze recordings we have the possibility to gain insights in previously unobservable cognitive processes involved in decision making. Thereby, understanding the connection between the observable single fixation and the respective unobservable processing of the attended information is of utterly importance. Evidence from 
the area of language processing and problem solving shows that the location and the duration of a fixation is strongly related in the sense that "the most active location in working memory will eventually determine the most likely direction of the eye movement at a given point in time" (Huettig et al, 2011). Various experimental studies have shown that people tend to direct their attention at what they are currently talking and thinking about (e.g., Griffin and Bock, 2000; Renkewitz and Jahn, 2012). Additional support for this assumption is given by the result that the sensitivity to stimuli is greater when the stimuli are presented at a location to which attention has been allocated (e.g., Bashinski and Bacharach, 1980; Reynolds et al, 2000). The recent formulation of the Attentional Drift Diffusion Model (Krajbich et al, 2010) in the area of computational neuroscience researchers become even more concrete and claim that the proportion of attention to a particular option is strongly predictive for subsequent choice behavior (see also work by Shimojo et al, 2003).

Not only attention towards alternatives in general, but also towards specific attributes of an alternative has been shown to be predicted by the importance and weighting of a specific piece of information in the decision making process (e.g., Fiedler et al, 2013; Reisen et al, 2008).

Building on this assumption we will use eye-tracking to investigate the relative weighting and importance of attributes in the decision making process. Additionally we will provide insights in how this weighting changes through different frames. 


\section{Theoretical Framework}

\subsection{Setup}

Our aim is to understand framing effects in social interactions. We focus on binary choice without uncertainty and without strategic interaction. Specifically, we look at a modified dictator game setting (Andreoni and Miller, 2002) in which subjects choose between two distributions of money between themselves and another anonymous participant. One option will be called the "selfish" option and the other the "altruistic" option.

Let the "selfish" option be denoted as $\left(s_{i}, s_{j}\right)$, where $s_{i}$ determines the own outcome and $s_{j}$ the outcome of the other player. Then, the "altruistic" option is given by $\left(a_{i}, a_{j}\right)=\left(s_{i}-c, s_{j}+b\right)$, where $a_{i}$ is the own outcome and $a_{j}$ is the other player's outcome. We set $c>0$ and $b>c$, such that the altruistic option will always be socially efficient. Further, we vary two types of situations. Either, subjects are ahead of their partner in both options $\left(a_{i}>a_{j}\right.$ and $\left.s_{i}>s_{j}\right)$ or they are behind regarding the outcomes $\left(a_{i}<a_{j}\right.$ and $\left.s_{i}<s_{j}\right)$.

We introduce a framing manipulation by presenting the same allocation either as a gain (GAIN) or as a loss (LOSS). Let $e_{G}$ be the endowment in GAIN and $e_{L}$ the endowment in LOSS (with $e_{L}>e_{G}$ ). Then, the two options can be described in terms of gains $(g)$ and losses $(l)$. Specifically, the selfish option $\left(s_{i}, s_{j}\right)$ can be expressed as $\left(s_{i}=e_{G}+g_{i}, s_{j}=e_{G}+g_{j}\right)$ in GAIN and as $\left(s_{i}=e_{L}-l_{i}, s_{j}=e_{L}-l_{j}\right)$ in LOSS. The altruistic option is defined accordingly. Importantly, both framing manipulations are identical from a payoff perspective. 


\subsection{Reference Dependent Altruism}

In this section we discuss requirements on the theory. We are interested in models dealing with context effects and with gain-loss framing in particular. We are interested in a theory which gives us a unified account for different context effects. Specifically, we look for a theory which endogenously predicts whether subjects choose the altruistic, efficient option or whether they choose the selfish option. Further, we are interested in predictions regarding process data. In particular, we want to predict relative attention to certain information and decision times.

Note that we are not interested in comparing and testing different theories of fairness even though eye-tracking might be a powerful tool to do this. Rather, we are interested in whether framing influences attentional processes. For this we employ a parsimonious model which is easily applicable.

There is little evidence on what to expect in regard of a shift in attentional processes. To the best of our knowledge there is no single model that could predict choices, decision time, and relative proportion of fixations at the same time. Our approach is to use a behavioral model, interpret it as a process model and use it to make predictions about the choice and information search behavior.

We assume that attention is guided by the importance of certain attributes of the situation to the decision maker. Therefore, the model needs to predict the relative weight of the attributes that guide the choice. To simplify, we reduce them for now to two simple attributes: own payoff and recipient payoff. Accordingly, for our setting the model would need to feature weights for the own income in relation to the other player's income. 
We focus on one specific theory, namely Reference Dependent Altruism (RDA, Breitmoser and Tan, 2013, 2014) which fits our requirements.

The basic idea of RDA is that agents are more altruistic when their income is above a certain reference point. The authors focus on two types of reference points: The absolute reference point is determined by an endowment or by the ex-ante expected payoff $\left(x_{i}^{*}\right)$. The relative reference point is determined by another agent's payoff $\left(x_{j}\right)$.

RDA suggests a strong difference in behavior when their income is higher than that of the other subject, i.e. they are ahead of the other subject in terms of payoff compared to being behind. Subjects should be more altruistic when they are ahead. Also, when subjects are above their absolute reference point, e.g., by experiencing a gain, they are assumed to be more altruistic compared to when facing losses. For our purposes we induce gains and losses through the endowment and assume that the endowment determines the absolute reference point. The decision maker's income in GAIN (LOSS) is therefore always above (below) the absolute reference point ${ }^{1}$

In our setup subjects always face a relative reference point (the other player's payoff $x_{j}$ ) and an absolute reference point $x^{*}$ at the same time. The combination of the frame (GAIN, LOSS) and relative payoffs (ahead, behind) leads to four situations (GAIN-ahead, GAIN-behind, LOSS-ahead, LOSS-behind). We obtain the following utility function $U_{i}=x_{i}+\alpha_{i}(F, R) x_{j}$ where $\alpha_{i}$ now depends on the absolute reference point determined by frame F (with $F \in\{G A I N, L O S S\}$ ) and on the relative reference point $\mathrm{R}$ (with $R \in\{$ ahead, behind $\}$ ). Further, subjects are (potentially) heterogeneous in

\footnotetext{
${ }^{1}$ Our predictions hold as long as we assume that the absolute reference point in Loss is higher than in GAIN (compare Grolleau et al, 2014).
} 
their parameters. Following RDA, we assume $\alpha(\cdot$, ahead $)>\alpha(\cdot$, behind $)$ and $\alpha(G A I N, \cdot)>\alpha(L O S S, \cdot)$, i.e. the altruism parameter is higher when subjects' income is above their relative reference point (independent of the frame) and higher when subjects' income is above their absolute reference point (independent of the relative comparison). Consequently, an individual acting according to this model could be characterized by four altruism parameters, one for every situation. ${ }^{2}$ In order to compare the model predictions with individual behavior, we elicit these parameters for every subject in a pre-test (see Appendix 7.2).

\subsection{Choice predictions}

Given a choice between the selfish option $S=\left(s_{i}, s_{j}\right)$ and the altruistic option $A=\left(a_{i}, a_{j}\right)=\left(s_{i}-c, s_{j}+b\right)$ and a situation-dependent altruism parameter $\alpha$, a subject chooses the altruistic option (here option A) whenever:

$$
\begin{gathered}
U_{i}(A)=s_{i}-c+\alpha_{i}(F, R)\left(s_{j}+b\right)>U_{i}(S)=s_{i}+\alpha_{i}(S, R) s_{j} \\
\Leftrightarrow \alpha_{i}(F, R)>\frac{c}{b}
\end{gathered}
$$

Therefore, in the model, the choice is determined only by the costbenefit factor (the fraction $c / b$ ) of the specific decision and the altruism parameter. In the experiment the cost-benefit factor varies between options. Given the assumptions over the altruism parameters the following two hypotheses follow directly.

\footnotetext{
${ }^{2}$ Note that we make no assumptions about the interaction of absolute and relative reference points.
} 
HC1: Overall, subjects are more likely to choose the altruistic option in GAIN compared to LOSS. On an individual level, the choice is determined by the parameters collected in the online pre-test.

HC2: Subjects are more likely to choose the altruistic option when being ahead compared to being behind. On an individual level, the choice is determined by the parameters collected in the online pre-test.

\subsection{Predictions about attentional process}

In this section we derive predictions about the attentional process. In particular, we make prediction about the relative proportion of attention to own and other-regarding information as well as the extent of information search, i.e. the decision time. We interpret the RDA model as a process model. For this, we adapt the basic assumption that subjects fixate more on information which is more important for their decision. We then interpret the altruism parameter as a measure of relative importance of otherregarding information in comparison to self-regarding information. Given the above assumptions on the context-dependent parameters $\alpha(F, R)$ This provides us with the following hypothesis.

HA1: Information search behavior differs in terms of relative fixations to own and other income between treatments. Subjects in LOSS focus more on their own income compared to subjects in GAIN. On an individual level the relative proportion to the own outcome correlates negatively with the altruism parameter collected in the online pre-test.

HA2: Information search behavior differs in terms of relative fixations 
to own and other income between situations where subjects are ahead and where they are behind in their payoffs. On an individual level the relative proportion to the own outcome correlates negatively with the altruism parameter collected in the online pre-test.

In order to get insights into whether framing makes a computational difference, we look at a measure for the depth of information, in particular the overall number of fixations and decision time. Following up on our process interpretation of the RDA model and the above assumptions we do not expect an influence of the frame and the relative income on the decision time. More precisely, our adapted RDA model only allows us to make predictions about the relative importance of information and not about a general difference between loss and gain decisions.

HA3: The absolute number of fixations and decision time does not vary between frames and relative income situations.

\section{Experimental Design}

\subsection{Laboratory setup}

Subjects in the experiment played multiple binary modified dictator games. In total, they faced 40 different decision tasks. For each of these decisions, subjects had to choose between a selfish option and an altruistic option. All options, however, gave both the decision maker and the receiver positive payoffs. The options were designed such that the altruistic option was additionally the socially efficient option, maximizing total payoffs. Subjects choosing the altruistic option had to forgo payoffs when choosing the 
altruistic option compared to the selfish option.

Framing was induced by describing each option pair either as a gain (GAIN treatment) or a loss (LOSS treatment). Subjects took part in only one treatment in a between-subjects design. To accomplish identical final payoffs in both treatments subjects received an endowment of $0.25 €$ in GAIN and $9 €$ in LOSS. The endowment was made particularly salient by handing out the endowments before subjects started their task. ${ }^{3}$ Additionally, in half of the decisions subjects earned more than their counterpart (irrespective of their choice) whereas in the other half of the decisions subjects earned less. We denote these decisions as ahead and behind, respectively.

Each subject faced the same items. The specific items were generated randomly within a given set of parameters. Specifically, the cost-benefit ratio of the generated mini dictator games was uniformly distributed between 0.1 and $0.9 .^{4}$ Also, the screen position (left vs. right) of the altruistic and selfish options were counterbalanced. The specific set of items used for this study can be found in Appendix 7.1. To control for order effects we varied the order in which the items appeared randomly for each subject. Subjects received detailed instructions and answered control questions about the nature of the game before they started (find the full set of instructions in appendix). Subjects were informed that one randomly selected item would be played out and become payoff relevant for themselves and their matched counterpart..$^{5}$

\footnotetext{
${ }^{3}$ Money was put in a cup labeled with the amount that it was containing and placed right next to the subject for the time of the experiment.

${ }^{4}$ At the same time average payoffs, sums and differences over all games are identical (with a margin of $0.1 €$ ) between the left and right option.

${ }^{5}$ After finishing the 40 decision tasks, subjects also took part in the counterfactual treatment. Subjects were aware that there was a second part involving some decisions but had no further information. Only one choice of both these parts was paid. The specific decision was only revealed in the very end. In this study we focus on the
} 
Additionally, subjects took part in an incentivized online pre-test (at least 24 hours before the experiment) where we elicited their altruism parameters for the four situations (GAIN-ahead GAIN-behind, LOSS-ahead, LOSS-behind) using multiple choice lists. This way, we obtain four situationdependent altruism parameters for each subject (the choice lists and a more detailed description of the procedure can be found in Appendix 7.2).

\subsection{Decision screens and eye-tracking}

In addition to choices we recorded subject's gaze behavior. Each decision started with a blank screen (3000ms), followed by a fixation cross $(500 \mathrm{~ms})$ before subjects had to decide about two simultaneously presented outcome allocations (Figure 1). The two options differed on the dimension of own, other, differences, and sum of outcomes. All of this information was presented on the screen to avoid any need for calculation and making the information processing easily observable.

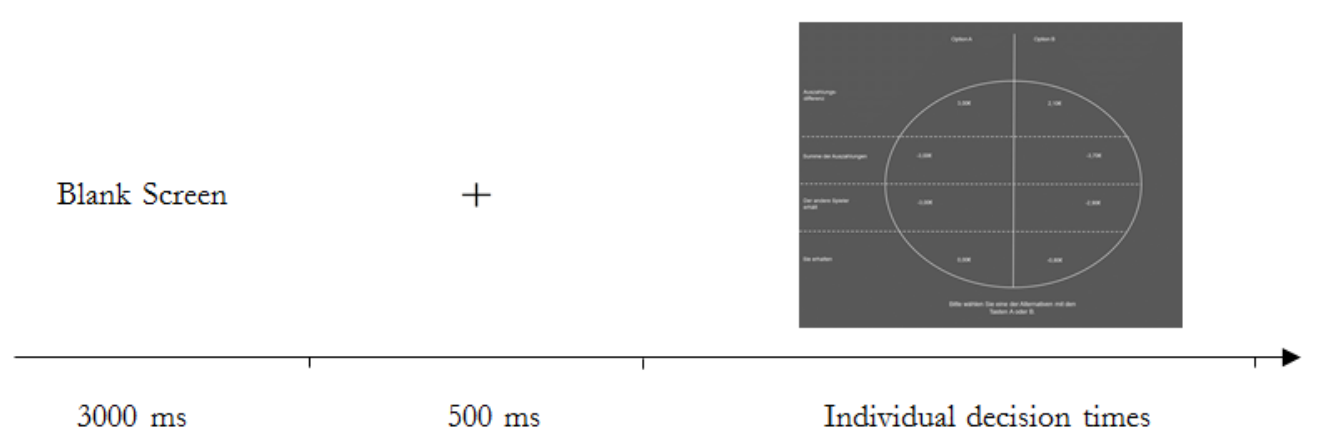

Figure 1: Sequence for each trial

Figure 2 shows a schematic version of the decision screen. The location

first part of the study since this provides us with the cleanest data. The data of the counterfactual is available on request. 
of the information (e.g. which information was presented on the top) varied between subjects but was constant over all trials for each individual subject.

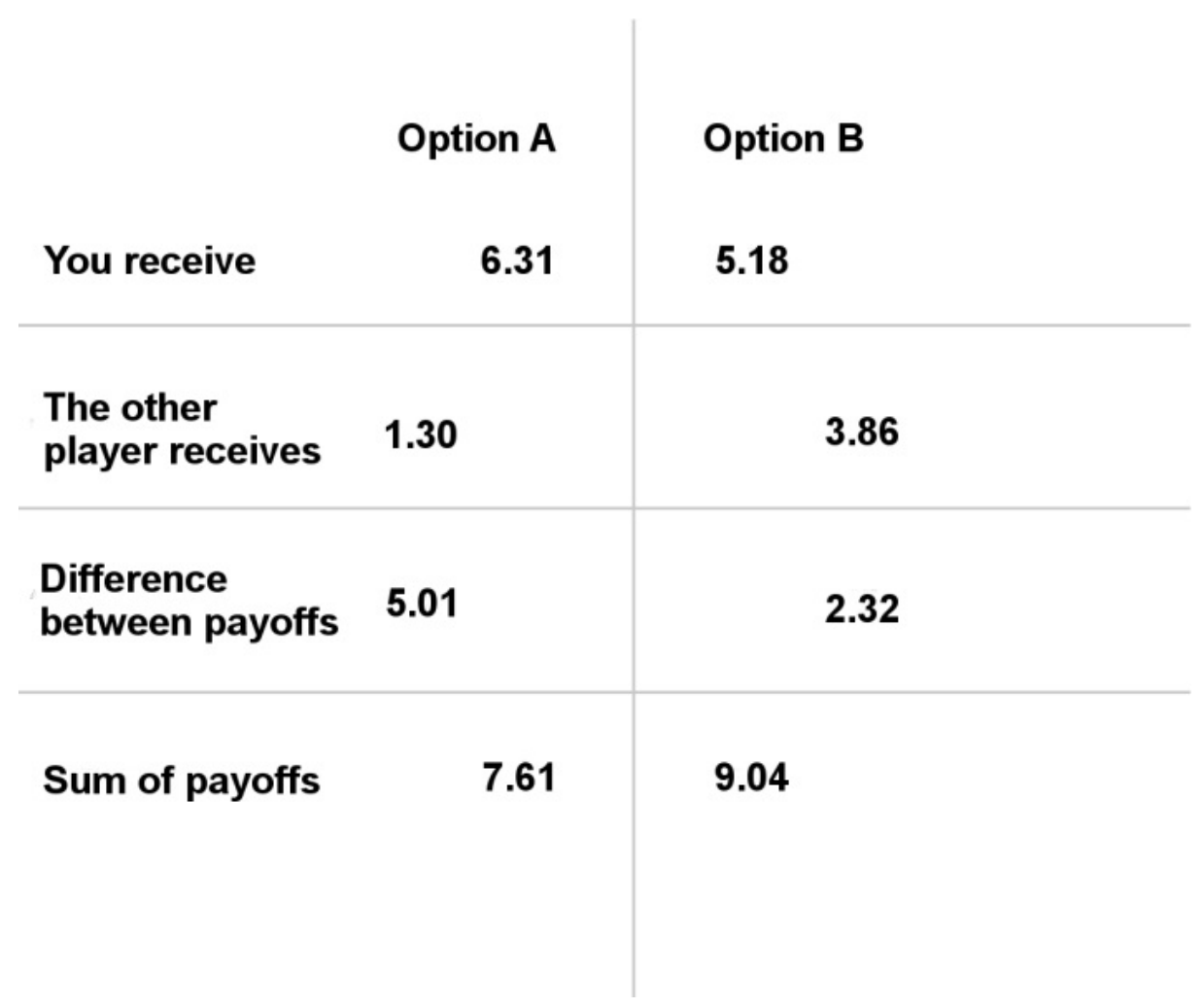

Figure 2: Decision screen (schematic)

Eye movements were recorded using three Eyegaze binocular remote systems with sampling rate of $120 \mathrm{~Hz}$ and an accuracy of about 0.45 degree. Tasks were presented at screen with a display resolution of 1280 x 1024 pixels. To secure data quality a chin rest mounted $60 \mathrm{~cm}$ away from the screen was used to minimize head movements. Participants indicated their choices by key press ("C" for the option on the left and "M" for the option on the right). The Experiment was run in Presentation ${ }^{\circledR}$.

For the analysis eight non-overlapping 100 x 100 pixels large areas of interest (AOIs) were defined. All 8 AOIs contained payoff information ( 2 own payoffs, 2 other players payoffs, 2 differences in payoffs, 2 sum in 
payoffs). Additional AOIs (100 x 190 pixels) containing content labels as well as the information about Options ("A" and "B") were defined to check if the trials pass the common quality thresholds, but were not used for the following analysis. The recorded choices as well as the eye-tracking data were pre-processed via Stata13.

Fixations were defined as periods of relative stable gazes within an area of 30 pixel. Fixations shorter than $50 \mathrm{~ms}$ were excluded from the analysis. Decisions made faster than $200 \mathrm{~ms}$ as well as tasks with duration longer than 3 standard deviations from the mean (within the particular decision block) were excluded from the analysis. In total $11.59 \%$ of recorded fixations were excluded this way. We analysed the number of fixations in each AOI and the decision times.

\subsection{Procedure}

In total 87 individuals, 44 in LOSS and 43 in GAIN (overall $60 \%$ were female), were recruited from the MPI Decision Lab Subject pool using Orsee (Greiner, 2015). All subjects had normal or corrected-to-normal vision. The experiment was conducted in September 2014 at the MPI Decision Lab in Bonn. The task in the lab took on average 30 minutes and subjects earned on average a total of $8.63 €$.

\section{Results}

In this chapter, we test our hypothesis and provide additional results. First, we present choice data and afterwards show the results of the process data. 


\subsection{Choices}

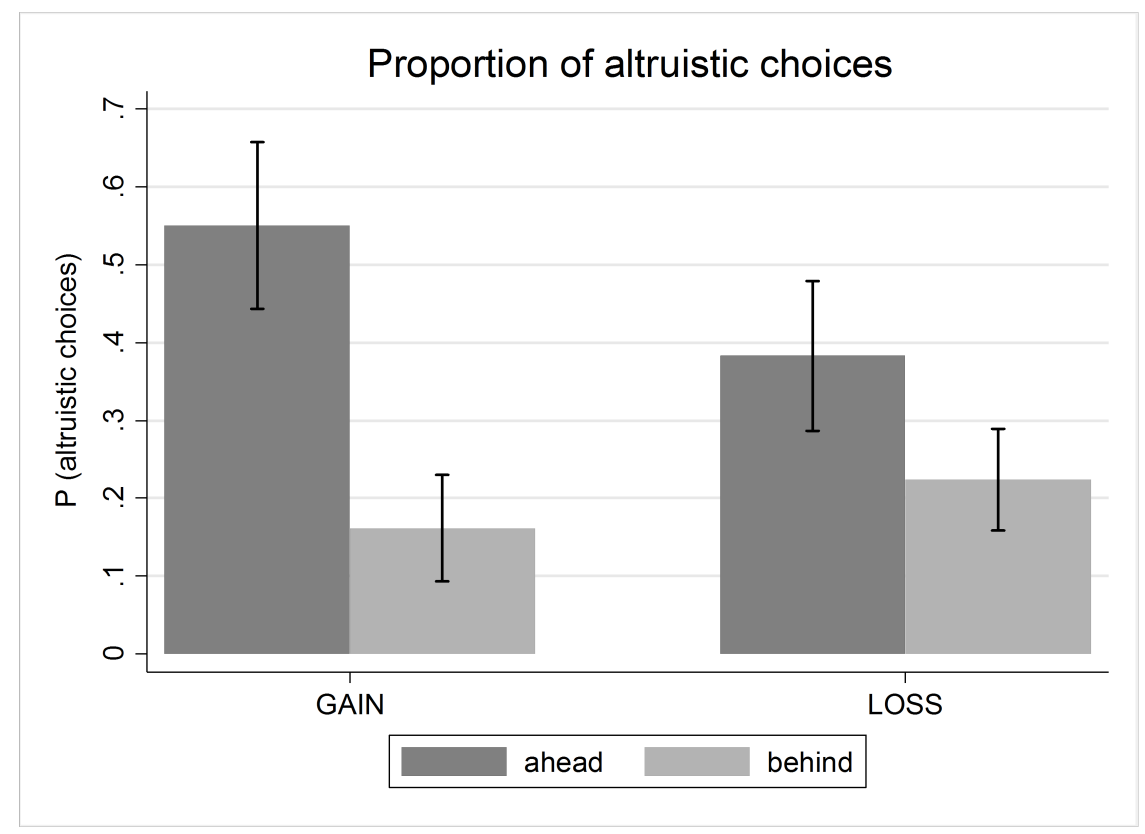

Figure 3: Altruistic choices by frame and relative income

Figure 3 shows the share of altruistic choices in both treatments split for situations where decision makers are ahead or behind, i.e., when their income is above or below the income of the other subject. We find a clear framing effect when subjects are ahead of the other player with subjects in GAIN choosing the altruistic option more often compared to subjects in LOSS. (55\% vs. 38\%). Using a conservative measure treating individual mean cooperation rates as observations $(\mathrm{N}=87)$, we find that this difference is significant (rank-sum test, $p=0.027$ ).

Result 1. Subjects are more likely to choose the altruistic option in the gain-frame compared to the loss-frame when their income is above the income of the receiver.

No such difference was found for situations in which the participant had 
lower payoffs than her matched player (behind). If anything the loss frame induced participants to be less altruistic in the gain frame compared to the loss frame ( $16 \%$ vs. $22 \%$, rank-sum test, $\mathrm{p}=0.183$ ). Additionally, these results show that being ahead leads to a higher share of altruistic choices independent of the frame (GAIN and LOSS, signed-rank test, $p<0.001$ ).

Result 2. Subjects are more likely to choose the altruistic option when their income is higher than the income of the receiver compared to situations with lower relative income.

On an individual level, our hypothesis state that the individual parameters as elicited in the online pre-test should predict behaviour in the experiment. In total $77 \%$ of all choices are predicted correctly this way, i.e., using the RDA model with the elicited parameters leads to a higher utility for the chosen option.

As a further factor for the altruistic choice, the cost-benefit is be important. As we described in the theory section, a higher cost-benefit ratio should lead to overall less altruistic choices. The following random-effects logit regression (table 1) shows that this is indeed the case. The main treatment effect for cases where subjects are ahead is robust against this control also when including the interaction between treatment and cost-benefit factor as well as the interaction term and order effects. Interestingly, for behind the coefficient for the cost-benefit factor is larger, i.e., subjects react more towards the efficiency of the altruistic choice. 
Table 1: Altruistic choices

\begin{tabular}{lcccccc}
\hline & $(1)$ & $(2)$ & $(3)$ & $(4)$ & $(5)$ & $(6)$ \\
& ahead & ahead & ahead & behind & behind & behind \\
\hline LOSS & $-1.600^{*}$ & $-1.447^{+}$ & $-1.472^{+}$ & 0.813 & $1.149^{+}$ & 1.138 \\
& $(0.692)$ & $(0.760)$ & $(0.762)$ & $(0.626)$ & $(0.698)$ & $(0.699)$ \\
Cost-benefit & $-4.665^{* * *}$ & $-4.494^{* * *}$ & $-4.548^{* * *}$ & $-6.714^{* * *}$ & $-6.076^{* * *}$ & $-6.113^{* * *}$ \\
& $(0.361)$ & $(0.502)$ & $(0.506)$ & $(0.522)$ & $(0.772)$ & $(0.774)$ \\
Interaction & & -0.341 & -0.272 & & -1.092 & -1.052 \\
& & $(0.706)$ & $(0.709)$ & & $(1.026)$ & $(1.027)$ \\
Order effects & & & YES & & & YES \\
& & & & & & \\
Constant & $2.573^{* * *}$ & $2.491^{* * *}$ & $2.716^{* * *}$ & -0.655 & -0.842 & $-1.039^{+}$ \\
& $(0.522)$ & $(0.547)$ & $(0.568)$ & $(0.487)$ & $(0.515)$ & $(0.539)$ \\
& & & & & & \\
\hline Subjects & 87 & 87 & 87 & 87 & 87 & 87 \\
Observations & 1740 & 1740 & 1740 & 1740 & 1740 & 1740 \\
\hline
\end{tabular}

This table shows results from a random-effects logit regression. The dependent variable is 1 for the altruistic choice and 0 for the selfish choice. LOSS is a dummy taking 1 in the LOSS treatment and 0 in the GAIN treatment. "Cost-benefit" ranges between 0.1 and 0.9. "Interaction" is the interaction term between the treatment and the cost-benefit factor. "Order effects" controls for the trial order. Standard errors are in parentheses. $+p<.10,{ }^{*} p<.05,{ }^{* *} p<.01,{ }^{* * *} p<.001$

\section{$5.2 \quad$ Information search process}

In order to explore whether and how framing influences the attentional process, we explore the information search behavior during the decision making process. In particular we examine the proportion of fixations to specific areas of interest (AOI, i.e., own gains / losses, other players gains / losses, difference between gains / losses and the sum of the own and the other players gains / losses), the overall number of fixations, and decision times per trial. The proportion of fixations to a specific AOI will give us insights on the relative weight that subjects put on this information. On 
the other hand, the absolute number of fixations and the decision time is an indicator for the depth of the information search and gives additional information on the complexity of the decisions.

\subsection{Proportion of attention to AOIs}

The proportion of attention is defined as the relative number of fixations to a specific AOI in relation to the total number of fixations on all AOIs in each trial. Figure 4 shows the average proportion of fixations to the specific AOI in both frames.

Overall, subjects attend their own payoffs more (57\%) than all the other information combined with the other player's payoff being the second most fixated information (26\%), the difference between payoffs being next (10\%) and the sum of payoffs being the least fixated information (7\%).

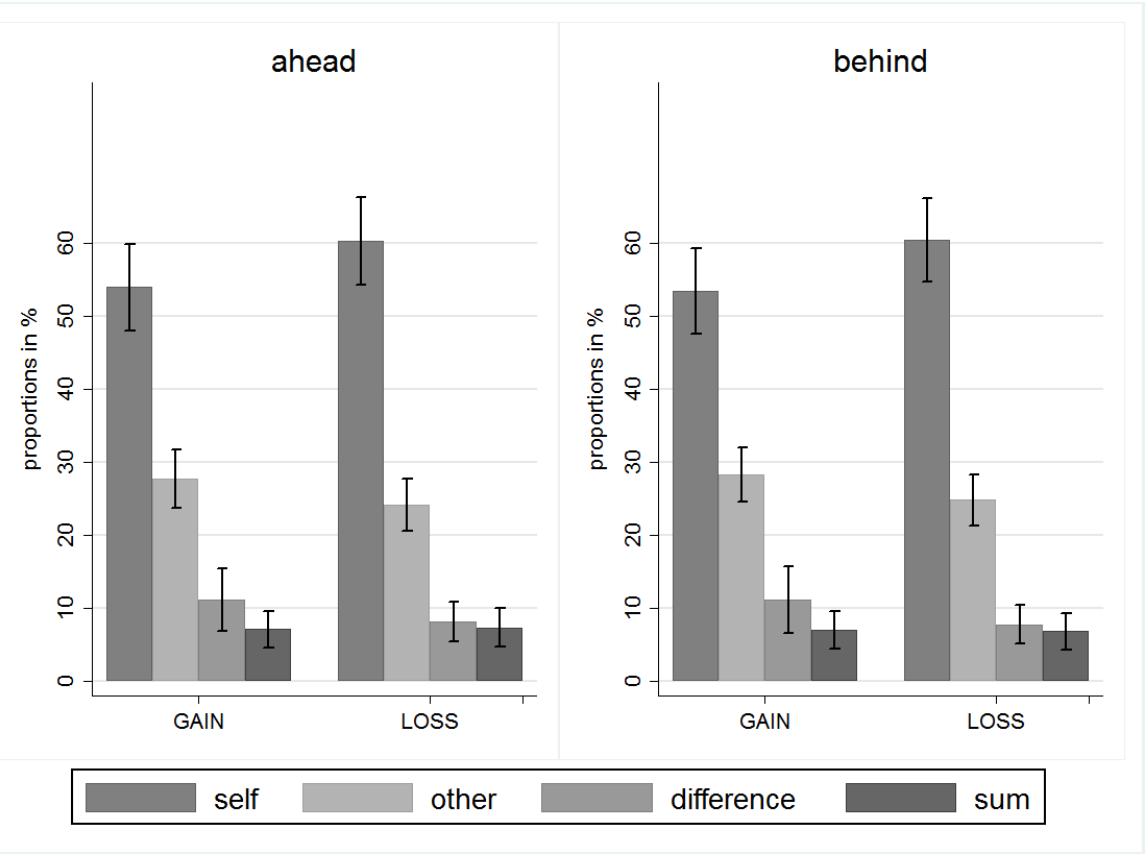

Figure 4: Proportion of Attention to AOIs

Our data shows a clear difference in the information search behaviour 
between the two frames. We treat the average proportion of attention for each subject as one observation and find that subjects in GAIN fixate less on their own payoff than subjects in LOSS $(53.7 \%$ vs. $60.4 \%$, rank-sum test, $p=0.014)$. The same holds true when restricting observations to ahead (54.0\% vs. $60.3 \%$, rank-sum test, $p=0.0717)$ and behind (53.4\% vs. $60.5 \%$, rank-sum test, $p=0.0880)$.

Result 3.In the loss-frame subjects fixate relatively more on own outcomes compared to the gain-frame.

But, there is no overall significant difference in the proportion of attention to own payoffs in situations where subjects are ahead compared to when they are behind $(57.1 \%$ vs $57.0 \%$, sign-rank test, $p=0.9571)$. The proportion of attention is also not significantly different between ahead and behind when restricting observation to the GAIN treatment (54.0\% vs. $53.4 \%$, sign-rank test, $p=0.8374$ ) or the LOSS treatment (60.3\% vs. $60.5 \%$, sign-rank test, $p=0.8848)$.

Result 4. The relative income does not influence the proportion of attention to the own outcome.

On an individual level, we hypothesized that subjects with stronger altruistic preferences weight own payoffs in the information search process less than people with more individualistic preferences. For the analysis we first look at each situation separately and correlate individual averages of the proportion of attention to the own outcome with the situationdependent altruism parameter. We get a negative and significant correlation for GAIN-ahead (Spearman's, $\rho=-0.5455, p<0.001$ ), GAIN-behind (Spearman's, $\rho=-0.6443, p<0.001$ ) and LOSS-behind (Spearman's, 
$\rho=-0.4961, p<0.001)$. For LOSS-ahead the correlation is also negative but not significant (Spearman's, $\rho=-0.2488, p=0.1076)$. Additionally, pooling all four situations and using the average of the four elicited altruism parameters for each subject we also find a negative and significant correlation (Spearman's, $\rho=-0.6509, p<0.001$ ).

Result 5. More altruistic subjects fixate relatively less on their own outcome.

\subsection{Extent of information search}

We use the absolute number of fixations as indicators for the depth of information search. Since fixations are very stable with respect to their duration this relates directly to the decision time. For the absolute number of fixations we count all fixations located within the predefined AOIs containing payoff information. ${ }^{6}$

The average total number of fixations does not differ between treatments (GAIN: 19.7 LOSS: 20.1, rank-sum test $p=0.8833$ ). The same holds true when restricting for situations where subjects are ahead (20.1 vs. 19.4 fixations, rank-sum test, $p=0.9312$ ) and behind (20.0 vs. 20.2 fixations, rank-sum test, $p=0.5775)$.

Similarly, the decision time does not vary significantly between treatments (GAIN: 5.34s LOSS: 5.56s, rank-sum test $p=0.7265$ ). Again, restricting for cases where subjects are ahead or behind does not influence this difference (ahead: 5.41s vs. 5.28s, rank-sum test, $p=0.9759$; behind: $5.50 \mathrm{~s}$ vs. $6.61 \mathrm{~s}, p=0.4550)$.

\footnotetext{
${ }^{6}$ Also remember, that we excluded fixations to text-AOIs from the analysis. The number of fixations is thus the number of fixations to value information only.
} 
Finally, subjects do not gather more information or have longer decision times when they are ahead compared to being behind (absolute number of fixations and decision time in both frames, sign-rank test, $p>0.1$ )

Result 6. Decision times and the absolute number of fixations are not influenced by the frame. Further, the relative income does not influence the extent of information search.

\section{Discussion and Conclusion}

In this paper, we studied the influence of gain-loss framing on behavior in interdependent social decisions. We observed subjects' information search behavior using eye-tracking. In our lab experiment we find that subjects facing a gain-frame are more likely to choose the altruistic option compared to subjects facing a loss frame if their payoffs are higher than the payoffs of the receiver. In cases where the payoff of the decision maker is lower than that of the receiver subjects are overall less altruistic but we do not find a difference between frames. Concerning the process data, we find that subjects in the loss frame fixate relatively more on their own income compared to subjects in the gain frame independently of whether their income is above or below that of the other subject.

Eye-tracking in combination with the choice data provides us with a rich data set. This allows us to analyse the underlying mechanism behind choices. The process data suggests that subjects that face losses weight their own payoffs higher in relation to the receiver's payoff compared to subjects that face gains. In effect this means that subjects experience loss aversion more in the own-outcome domain compared to the other-outcome 
domain, i.e., they put a higher weight on the losses to their own outcome compared to losses of the other players outcome. This interpretation of the results is in line with the notion of impure altruism or warm-glow giving (Andreoni, 1990). Subjects mainly care about giving some amount but not necessarily about the utility of the receiver.

We employed a model of reference dependent altruism (Breitmoser and Tan, 2014) and interpret it as a process model. The model, in combination with parameters elicited before the experiment, proved to be capable of predicting $77 \%$ of subject's choices in our experiment correctly. Importantly, the eye-tracking data allows us to evaluate the model's assumptions. Subjects facing payoffs below their absolute reference point (loss-frame) focus their attention more on their own outcome compared to subjects with an income above their absolute reference point (gain-frame). This clear difference in information search behavior is in line with the assumption that the absolute reference point (here the endowment) influences altruistic preferences in a non-continuous way. Also, the proportion of attention to the other-regarding information directly correlates with the situationdependent altruism parameters that we elicited in the online pre-test.

In contrast, we find a clear difference in choices between situations where subjects are ahead of others compared to situations where they are behind in payoffs but at the same time there is no difference in the information search behavior. This suggests that there is a more fundamental difference between an absolute reference point (e.g., the endowment) and a relative reference point (e.g., payoffs of the other subject). This deserves further exploration in the light of models of reference dependent preferences (Köszegi and Rabin, 2006; Schwerter, 2013). 
Finally, our results might also be interpreted as indication for an alternative attention-based channel through which framing influences choice. Previous results suggest that framing influences attentional processes (Kovach et al, 2014). At the same time, results from neuroscience show that manipulating attention might influence choice (Armel et al, 2008). Taken together, this provides the basis for a different, reversed mechanism: Framing manipulates which information decision makers attend to and this influences the decisions they make (see also Shimojo et al, 2003). When we interpret our results in this way, the proportion of attention would not be a reflection of the underlying weighting function but instead is driven by a preference for information (Falk and Zimmermann, 2014). For example, an altruistic subject is quite interested in another subject's payoffs while a purely selfish subject does not care at all about this information. This interest could then in principle be biased by the context or frame. In our context, losses would draw attention to the own income and away from the income of others. Then this would lead to an "attentional bias" towards the own outcome which would prompt subjects to make more selfish choices. The direction of the mechanism is still unclear. Does framing influence preferences which then drive the information search behavior? Or are preferences rather constructed and therefore prone to be influenced by an attentional bias? In order to test this one would have to manipulate attention directly (e.g., by highlighting some information on the decision screen) and see whether this influences choices. This provides a natural and important next step for our research.

The main goal of the paper is to improve the understanding of framing effects in social interactions. In this paper we demonstrate that eye-tracking 
data complements choice data in providing a rich data set and a powerful tool to improve and test assumptions of theoretical models. Therefore, our paper contributes to the goal to develop "a more unified approach toward decision-making" (Krajbich et al, 2014). 


\section{References}

Amos Tversky DK (1986) Rational choice and the framing of decisions. The Journal of Business 59(4):S251-S278, URL http://www.jstor.org/stable/2352759

Andreoni J (1990) Impure altruism and donations to public goods: A theory of warm-glow giving. The Economic Journal 100(401):464-477, DOI $10.2307 / 2234133$

Andreoni J, Miller J (2002) Giving according to garp: An experimental test of the consistency of preferences for altruism. Econometrica 70(2):737753

Antinyan A (2014) Loss and other-regarding preferences: Evidence from dictator game. Department of Management, Università Ca'Foscari Venezia Working Paper 3

Arieli A, Ben-Ami Y, Rubinstein A (2009) Fairness motivations and procedures of choice between lotteries as revealed through eye movements. Eitan Berglas School of Economics

Arieli A, Ben-Ami Y, Rubinstein A (2011) Tracking decision makers under uncertainty. American Economic Journal: Microeconomics 3(4):68-76

Armel KC, Beaumel A, Rangel A (2008) Biasing simple choices by manipulating relative visual attention. Judgment and Decision Making 3(5):396

Balcombe K, Fraser I, Williams L, McSorley E (2017) Examining the relationship between visual attention and stated preferences: A discrete 
choice experiment using eye-tracking. Journal of Economic Behavior \& Organization 144:238-257

Bashinski HS, Bacharach VR (1980) Enhancement of perceptual sensitivity as the result of selectively attending to spatial locations. Perception \& psychophysics 28(3):241-248

Baumeister RF, Bratslavsky E, Finkenauer C, Vohs KD (2001) Bad is stronger than good. Review of General Psychology 5(4):323

Bernheim BD, Rangel A (2007) Toward choice-theoretic foundations for behavioral welfare economics. The American economic review 97(2):464470

Breitmoser Y, Tan JH (2013) Reference dependent altruism in demand bargaining. Journal of Economic Behavior \& Organization 92:127-140, DOI http://dx.doi.org/10.1016/j.jebo.2013.06.001, URL http://www.sciencedirect.com/science/article/pii/S0167268113001509

Breitmoser Y, Tan JH (2014) Reference dependent altruism. mimeo

Brekke KA, Konow J, Nyborg K (2012) Cooperation is relative: Income and framing effects with public goods

Buchan N, Croson R, Johnson E, Wu G (2005) Gain and loss ultimatums. Advances in Applied Microeconomics 13:1-23

Camerer C, Loewenstein G (2004) Behavioral economics: Past, present, future. Princeton: Princeton University Press 
Chen CT, Huang CY, Wang JTy (2018) A window of cognition: Eyetracking the reasoning process in spatial beauty contest games. Games and Economic Behavior

Dariel A (2013) Cooperation preferences and framing effects. Discussion Papers, Department of Economics, Universität Bern

De Dreu CKW (1996) Gain-loss-frame in outcome-interdependence: does it influence equality or equity considerations? European Journal of Social Psychology 26(2):315-324

De Dreu CKW, Lualhati JC, McCusker C (1994) Effects of gain-loss frames on satisfaction with self-other outcome-differences. European Journal of Social Psychology 24(4):497-510

Dehue FMJ, McClintock CG, Liebrand WBG (1993) Social value related response latencies: Unobtrusive evidence for individual differences in information processing. European Journal of Social Psychology 23(3):273293

Devetag G, Guida S, Polonio L (2015) An eye-tracking study of featurebased choice in one-shot games. Experimental Economics 19(1):177-201

Dohmen T, Falk A, Fliessbach K, Sunde U, Weber B (2011) Relative versus absolute income, joy of winning, and gender: Brain imaging evidence. Journal of Public Economics 95(3):279-285

Dufwenberg M, Gächter S, Hennig-Schmidt H (2011) The framing of games and the psychology of play. Games and Economic Behavior 73(2):459-478 
Ellingsen T, Johannesson M, Mollerstrom J, Munkhammar S (2012) Social framing effects: Preferences or beliefs? Games and Economic Behavior $76(1): 117-130$

Engel C, Rand DG (2014) What does "clean" really mean? the implicit framing of decontextualized experiments. Economics Letters 122(3):386389

Falk A, Zimmermann F (2014) Beliefs and utility: Experimental evidence on preferences for information. mimeo

Fehr E, Rangel A (2011) Neuroeconomic foundations of economic choicerecent advances. Journal of Economic Perspectives 25(4):3-30

Fiedler S, Glöckner A, Nicklisch A, Dickert S (2013) Social value orientation and information search in social dilemmas: An eye-tracking analysis. Organizational Behavior and Human Decision Processes 120(2):272-284

Greiner B (2015) Subject pool recruitment procedures: organizing experiments with orsee. Journal of the Economic Science Association 1(1):114125

Griffin ZM, Bock K (2000) What the eyes say about speaking. Psychological Science 11(4):274-279

Grolleau G, Kocher MG, Sutan A (2014) Cheating and loss aversion: do people lie more to avoid a loss? mimeo

Hochman G, Yechiam E (2011) Loss aversion in the eye and in the heart: The autonomic nervous system's responses to losses. Journal of Behavioral Decision Making 24(2):140-156 
Hochman G, Glöckner A, Yechiam E (2010) Physiological measures in identifying decision strategies. Foundations for tracing intuition: Challenges and methods pp 139-159

Huettig F, Olivers CN, Hartsuiker RJ (2011) Looking, language, and memory: Bridging research from the visual world and visual search paradigms. Acta Psychologica 137(2):138150, DOI http://dx.doi.org/10.1016/j.actpsy.2010.07.013, URL http://www.sciencedirect.com/science/article/pii/S0001691810001411

Kahneman D, Tversky A (1979) Prospect theory: An analysis of decision under risk. Econometrica: Journal of the Econometric Society pp 263291

Kahneman D, Tversky A (1984) Choices, values, and frames. American Psychologist 39(4):341

Kahneman D, Knetsch J, Thaler R (1990) Experimental tests of the endowment effect and the coase theorem. Journal of political Economy pp $1325-1348$

Kern MC, Chugh D (2009) Bounded ethicality the perils of loss framing. Psychological Science 20(3):378-384

Kühberger A (1998) The influence of framing on risky decisions: A meta-analysis. Organizational Behavior and Human Decision Processes $75(1): 23-55$

Kovach CK, Sutterer MJ, Rushia SN, Teriakidis A, Jenison RL (2014) Two systems drive attention to rewards. Name: Frontiers in Psychology 5:46 
Krajbich I, Dean M (2015) How can neuroscience inform economics? Current Opinion in Behavioral Sciences 5:51-57

Krajbich I, Rangel A (2011) Multialternative drift-diffusion model predicts the relationship between visual fixations and choice in value-based decisions. Proceedings of the National Academy of Sciences 108(33):13,85213,857

Krajbich I, Armel C, Rangel A (2010) Visual fixations and the computation and comparison of value in simple choice. Nature Neuroscience 13(10):1292-1298

Krajbich I, Oud B, Fehr E (2014) Benefits of neuroeconomic modeling: New policy interventions and predictors of preference. The American Economic Review 104(5):501-506

Krucien N, Ryan M, Hermens F (2017) Visual attention in multi-attributes choices: What can eye-tracking tell us? Journal of Economic Behavior \& Organization 135:251-267

Kőszegi B, Rabin M (2006) A model of reference-dependent preferences. The Quarterly Journal of Economics 121(4):1133-1165

Kuo FY, Hsu CW, Day RF (2009) An exploratory study of cognitive effort involved in decision under framing - an application of the eye-tracking technology. Decision Support Systems 48(1):81-91

Lahey JN, Oxley D (2016) The power of eye tracking in economics experiments. American Economic Review 106(5):309-13 
Leliveld MC, Beest Iv, Dijk Ev, Tenbrunsel AE (2009) Understanding the influence of outcome valence in bargaining: A study on fairness accessibility, norms, and behavior. Journal of Experimental Social Psychology $45(3): 505-514$

Levin IP, McElroy T, Gaeth GJ, Hedgcock W, Denburg NL (2014) Behavioral and neuroscience methods for studying neuroeconomic processes: What we can learn from framing effects, American Psychological Association, Washington, DC, US, pp 43-69. Bronfenbrenner series on the ecology of human development.

Liebrand WBG, McClintock CG (1988) The ring measure of social values: A computerized procedure for assessing individual differences in information processing and social value orientation. European Journal of Personality 2(3):217-230

Novemsky N, Kahneman D (2005) The boundaries of loss aversion. Journal of Marketing research 42(2):119-128

Polonio L, Di Guida S, Coricelli G (2015) Strategic sophistication and attention in games: An eye-tracking study. Games and Economic Behavior $94: 80-96$

Poppe M, Valkenberg H (2003) Effects of gain versus loss and certain versus probable outcomes on social value orientations. European Journal of Social Psychology 33(3):331-337

Reisen N, Hoffrage U, Mast FW (2008) Identifying decision strategies in a consumer choice situation. Judgment and Decision Making 3(8):641-658 
Renkewitz F, Jahn G (2012) Memory indexing: A novel method for tracing memory processes in complex cognitive tasks. Journal of Experimental Psychology: Learning, Memory, and Cognition 38(6):1622

Reutskaja E, Nagel R, Camerer CF, Rangel A (2011) Search dynamics in consumer choice under time pressure: An eye-tracking study. American Economic Review 101(2):900-926

Reynolds JH, Pasternak T, Desimone R (2000) Attention increases sensitivity of v4 neurons. Neuron 26(3):703-714

Salant Y, Rubinstein A (2008) (A, f): Choice with Frames. The Review of Economic Studies 75(4):1287-1296

Satterthwaite TD, Green L, Myerson J, Parker J, Ramaratnam M, Buckner RL (2007) Dissociable but inter-related systems of cognitive control and reward during decision making: Evidence from pupillometry and eventrelated fmri. Neuroimage 37(3):1017-1031

Schwerter F (2013) Social reference points and risk taking

Shimojo S, Simion C, Shimojo E, Scheier C (2003) Gaze bias both reflects and influences preference. Nature Neuroscience 6(12):1317-1322, DOI $10.1038 / \mathrm{nn} 1150$

Tversky A, Kahneman D (1981) The framing of decisions and the psychology of choice. Science 211(4481):453-458

Tversky A, Kahneman D (1991) Loss aversion in riskless choice: A reference-dependent model. The Quarterly Journal of Economics 106(4):1039-1061 
Wang JTy, Spezio M, Camerer CF (2010) Pinocchio's pupil: Using eyetracking and pupil dilation to understand truth telling and deception in sender-receiver games. American Economic Review 100(3):984-1007

Yechiam E, Hochman G (2013a) Loss-aversion or loss-attention: The impact of losses on cognitive performance. Cognitive Psychology 66(2):212231

Yechiam E, Hochman G (2013b) Losses as modulators of attention: Review and analysis of the unique effects of losses over gains. Psychological Bulletin 139(2):497

Zhou X, Wu Y (2011) Sharing losses and sharing gains: increased demand for fairness under adversity. Journal of Experimental Social Psychology $47(3): 582-588$ 


\section{Appendix}

\section{$7.1 \quad$ Items}

Table 2: Items in the experiment, numbers show final payoffs in $€$

\begin{tabular}{|l|lllll|l|ll|ll|}
\hline & \multicolumn{2}{|c|}{ Option A } & \multicolumn{2}{c|}{ Option B } & & \multicolumn{2}{c|}{ Option A } & \multicolumn{2}{c|}{ Option B } \\
\hline$\#$ & own & other & own & other & $\#$ & own & other & own & other \\
\hline 1 & 5,50 & 1,32 & 4,94 & 4,13 & 21 & 2,99 & 3,50 & 1,79 & 6,61 \\
2 & 4,64 & 4,06 & 5,15 & 1,41 & 22 & 3,25 & 4,36 & 2,64 & 6,13 \\
3 & 4,30 & 3,95 & 4,82 & 1,28 & 23 & 1,96 & 7,44 & 2,87 & 5,09 \\
4 & 5,08 & 4,32 & 5,62 & 1,52 & 24 & 3,23 & 5,15 & 3,76 & 4,06 \\
5 & 2,85 & 6,62 & 3,40 & 3,69 & 25 & 5,27 & 3,24 & 6,08 & 1,78 \\
6 & 2,68 & 3,47 & 2,10 & 7,11 & 26 & 7,08 & 1,44 & 5,54 & 3,65 \\
7 & 1,30 & 7,30 & 1,87 & 3,35 & 27 & 5,84 & 3,29 & 6,87 & 1,45 \\
8 & 1,37 & 7,67 & 2,10 & 3,92 & 28 & 6,37 & 1,91 & 5,72 & 3,03 \\
9 & 4,83 & 3,82 & 5,55 & 1,30 & 29 & 2,05 & 6,74 & 3,44 & 4,75 \\
10 & 5,49 & 1,38 & 4,85 & 4,51 & 30 & 1,29 & 5,29 & 1,9 & 4,10 \\
11 & 4,48 & 4,18 & 5,08 & 1,42 & 31 & 2,95 & 3,61 & 2,01 & 5,39 \\
12 & 4,69 & 4,32 & 5,24 & 1,79 & 32 & 1,46 & 7,52 & 3,34 & 4,60 \\
13 & 2,72 & 4,32 & 1,98 & 6,86 & 33 & 6,28 & 2,61 & 6,79 & 1,98 \\
14 & 2,75 & 4,35 & 2,22 & 6,96 & 34 & 5,03 & 1,67 & 3,58 & 3,32 \\
15 & 1,61 & 5,55 & 2,26 & 2,82 & 35 & 6,01 & 2,74 & 4,93 & 4,17 \\
16 & 2,89 & 3,27 & 1,83 & 7,43 & 36 & 6,26 & 2,95 & 6,81 & 2,29 \\
17 & 6,31 & 1,30 & 5,18 & 3,86 & 37 & 3,9 & 4,6 & 1,51 & 7,62 \\
18 & 5,70 & 2,45 & 5,04 & 3,78 & 38 & 3,45 & 5,55 & 1,52 & 7,96 \\
19 & 5,97 & 1,89 & 4,95 & 4,55 & 39 & 4,37 & 4,88 & 3,46 & 5,92 \\
20 & 4,33 & 1,87 & 3,76 & 3,30 & 40 & 2,29 & 4,52 & 1,70 & 5,28 \\
\hline
\end{tabular}




\subsection{Online pre-test}

Before the main experiment subjects took part in an online pre-test. Participants were faced with four multiple choice lists (MCL) as seen below. For each row they had to indicate whether they want to choose option A or option B. At the end of the experiment, one row from one randomly selected MCL was randomly selected and became payoff relevant for the decision maker and one other subject. The MCL were designed such that participants are expected to choose Option A for some rows and then switch to Option B for the remaining rows or, alternatively, choose Option B in all rounds. We choose this switching point as the point of indifference between Option A and Option B. This provides us with an altruism parameter for each MCL. Each MCL relates to one situation from the lab experiment, i.e., 2 MCLs feature decisions over gains and 2 MCLs feature decisions over losses. For gains and losses one table only has options where the subject earns more than the other subject and one where he earns less. All participants in the study were consistent in the sense that they indicated only one switching point for each MCL. 
Table 3: GAIN-ahead

\begin{tabular}{|c|c|c|}
\hline Option $\mathrm{A}$ & Option $\mathrm{B}$ & $\alpha$ for indifference \\
\hline$(1.90 € ; 0.20 €)$ & $(1.86 € ; 1.00 €)$ & 0.05 \\
$(1.90 € ; 0.20 €)$ & $(1.82 € ; 1.00 €)$ & 0.1 \\
$(1.90 € ; 0.20 €)$ & $(1.74 € ; 1.00 €)$ & 0.2 \\
$(1.90 € ; 0.20 €)$ & $(1.66 € ; 1.00 €)$ & 0.3 \\
$(1.90 € ; 0.20 €)$ & $(1.58 € ; 1.00 €)$ & 0.4 \\
$(1.90 € ; 0.20 €)$ & $(1.50 € ; 1.00 €)$ & 0.5 \\
$(1.90 € ; 0.20 €)$ & $(1.42 € ; 1.00 €)$ & 0.6 \\
$(1.90 € ; 0.20 €)$ & $(1.34 € ; 1.00 €)$ & 0.7 \\
$(1.90 € ; 0.20 €)$ & $(1.26 € ; 1.00 €)$ & 0.8 \\
$(1.90 € ; 0.20 €)$ & $(1.10 € ; 1.00 €)$ & 1 \\
\hline
\end{tabular}

Table 4: GAIN-behind

\begin{tabular}{|c|c|c|}
\hline Option $\mathrm{A}$ & Option $\mathrm{B}$ & $\alpha$ for indifference \\
\hline$(1.00 € ; 1.10 €)$ & $(0.96 € ; 1.90 €)$ & 0.05 \\
$(1.00 € ; 1.10 €)$ & $(0.92 € ; 1.90 €)$ & 0.1 \\
$(1.00 € ; 1.10 €)$ & $(0.84 € ; 1.90 €)$ & 0.2 \\
$(1.00 € ; 1.10 €)$ & $(0.76 € ; 1.90 €)$ & 0.3 \\
$(1.00 € ; 1.10 €)$ & $(0.68 € ; 1.90 €)$ & 0.4 \\
$(1.00 € ; 1.10 €)$ & $(0.60 € ; 1.90 €)$ & 0.5 \\
$(1.00 € ; 1.10 €)$ & $(0.52 € ; 1.90 €)$ & 0.6 \\
$(1.00 € ; 1.10 €)$ & $(0.44 € ; 1.90 €)$ & 0.7 \\
$(1.00 € ; 1.10 €)$ & $(0.36 € ; 1.90 €)$ & 0.8 \\
$(1.00 € ; 1.10 €)$ & $(0.20 € ; 1.90 €)$ & 1 \\
\hline
\end{tabular}


Table 5: LOSS-ahead

\begin{tabular}{|c|c|c|}
\hline Option $\mathrm{A}$ & Option $\mathrm{B}$ & $\alpha$ for indifference \\
\hline$(-0.20 € ;-1.90 €)$ & $(-0.24 € ;-1.10 €)$ & 0.05 \\
$(-0.20 € ;-1.90 €)$ & $(-0.28 € ;-1.10 €)$ & 0.1 \\
$(-0.20 € ;-1.90 €)$ & $(-0.36 € ;-1.10 €)$ & 0.2 \\
$(-0.20 € ;-1.90 €)$ & $(-0.44 € ;-1.10 €)$ & 0.3 \\
$(-0.20 € ;-1.90 €)$ & $(-0.52 € ;-1.10 €)$ & 0.4 \\
$(-0.20 € ;-1.90 €)$ & $(-0.60 € ;-1.10 €)$ & 0.5 \\
$(-0.20 € ;-1.90 €)$ & $(-0.68 € ;-1.10 €)$ & 0.6 \\
$(-0.20 € ;-1.90 €)$ & $(-0.76 € ;-1.10 €)$ & 0.7 \\
$(-0.20 € ;-1.90 €)$ & $(-0.84 € ;-1.10 €)$ & 0.8 \\
$(-0.20 € ;-1.90 €)$ & $(-1.00 € ;-1.10 €)$ & 1 \\
\hline
\end{tabular}

Table 6: LOSS-behind

\begin{tabular}{|c|c|c|}
\hline Option $\mathrm{A}$ & Option $\mathrm{B}$ & $\alpha$ for indifference \\
\hline$(-1.10 € ;-1.00 €)$ & $(-1.18 € ;-0.20 €)$ & 0.05 \\
$(-1.10 € ;-1.00 €)$ & $(-1.26 € ;-0.20 €)$ & 0.1 \\
$(-1.10 € ;-1.00 €)$ & $(-1.34 € ;-0.20 €)$ & 0.2 \\
$(-1.10 € ;-1.00 €)$ & $(-1.42 € ;-0.20 €)$ & 0.3 \\
$(-1.10 € ;-1.00 €)$ & $(-1.50 € ;-0.20 €)$ & 0.4 \\
$(-1.10 € ;-1.00 €)$ & $(-1.58 € ;-0.20 €)$ & 0.5 \\
$(-1.10 € ;-1.00 €)$ & $(-1.66 € ;-0.20 €)$ & 0.6 \\
$(-1.10 € ;-1.00 €)$ & $(-1.74 € ;-0.20 €)$ & 0.7 \\
$(-1.10 € ;-1.00 €)$ & $(-1.82 € ;-0.20 €)$ & 0.8 \\
$(-1.10 € ;-1.00 €)$ & $(-1.90 € ;-0.20 €)$ & 1 \\
\hline
\end{tabular}




\subsection{Instructions}

The original instructions in German are available from the authors upon request. Below is the English translation of the instructions used in treatment GAIN. Differences in the instructions in treatment LOSS are marked by square brackets "[...]".

\section{Information about the Experiment Welcome to the Experiment!}

Please read the following information carefully. In the instructions you will learn what you need in order to participate in the study. If you have any questions please indicate it. We will answer the question at your seat.

The study today consist of two parts. In each of the two parts you will make a series of decisions. At the end of today's session one of the decisions of one of the two parts will be selected. This decision will then be payoff relevant for you and another participant. You will receive your part of the payment at the end of today's sessions. The other participant will not be a participant of the current session but a participant of one of the following sessions. This participant will have the identical task and instructions as you.

Accordingly, for every other subject that took part in a session before your session, one of his decisions was selected for payment. You will receive, additionally to the payment based on your decision, a payment that is based on a decision that another participant in another session made. This participant will have the identical task and instructions as you.

Additionally, you will receive the payment of your online questionnaire. 
You will receive complete information about the decisions before the start of each part.

\section{Part 1}

In this part of the experiment you start with an endowment of $0.25 €$ [9€] which you find in the box in front of you.

In the following you will face a series of 40 decision task. In each of the tasks you need to decide how to split a gain [loss] between you and another randomly determined person. In each task you can choose between two options (left and right side) and thereby decide how to split gains [losses] between yourself and another person. Your decision is made by pressing the keys A and B marked in red. There are no right or wrong answers in this task.

After completion of the first part we will hand out information for the second part and store your endwoment until the payment.

Example:

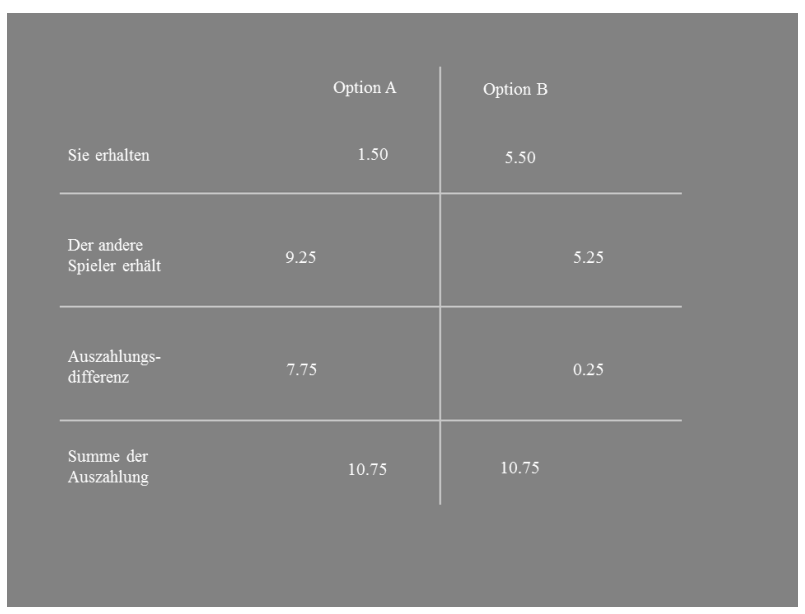

Figure 5: This is how the decision task will look like on the screen 